\title{
The effect of vertical and horizontal symmetry on memory for tactile patterns in late blind individuals
}

\author{
Zaira Cattaneo • Tomaso Vecchi - Micaela Fantino • \\ Andrew M. Herbert • Lotfi B. Merabet
}

Published online: 13 November 2012

(C) Psychonomic Society, Inc. 2012

\begin{abstract}
Visual stimuli that exhibit vertical symmetry are easier to remember than stimuli symmetric along other axes, an advantage that extends to the haptic modality as well. Critically, the vertical symmetry memory advantage has not been found in early blind individuals, despite their overall superior memory, as compared with sighted individuals, and the presence of an overall advantage for identifying symmetric over asymmetric patterns. The absence of the vertical axis memory advantage in the early blind may depend on their total lack of visual experience or on the effect of prolonged visual deprivation. To disentangle this issue, in this study, we measured the ability of late blind individuals to remember tactile spatial patterns that were either vertically or horizontally symmetric or asymmetric. Late blind participants showed better memory performance for symmetric patterns. An additional advantage for the vertical axis of symmetry over the horizontal one was reported, but only for patterns
\end{abstract}

\author{
Z. Cattaneo $(\bowtie)$ \\ Department of Psychology, University of Milano-Bicocca, \\ Milano, Italy \\ e-mail: zaira.cattaneo@unimib.it \\ Z. Cattaneo $\cdot$ T. Vecchi \\ Brain Connectivity Center, IRCCS Mondino, \\ Pavia, Italy \\ T. Vecchi $\cdot$ M. Fantino \\ Department of Psychology, University of Pavia, \\ Pavia, Italy \\ A. M. Herbert \\ Department of Psychology, Rochester Institute of Technology, \\ Rochester, NY, USA \\ L. B. Merabet \\ Department of Ophthalmology, Massachusetts Eye and Ear \\ Infirmary, Harvard Medical School, \\ Boston, USA
}

presented in the frontal plane. In the horizontal plane, no difference was observed between vertical and horizontal symmetric patterns, due to the latter being recalled particularly well. These results are discussed in terms of the influence of the spatial reference frame adopted during exploration. Overall, our data suggest that prior visual experience is sufficient to drive the vertical symmetry memory advantage, at least when an external reference frame based on geocentric cues (i.e., gravity) is adopted.

Keywords Symmetry $\cdot$ Tactile $\cdot$ Haptic $\cdot$ Late blind $\cdot$ Spatial memory $\cdot$ Reference frame

\section{Introduction}

Symmetry has been found to aid visual spatial memory for patterns, even when the task does not direct attention to that property, with an additional advantage for the vertical axis of symmetry over other orientations (Rossi-Arnaud, Pieroni, \& Baddeley, 2006, Rossi-Arnaud, Pieroni, Spataro, \& Baddeley, 2012). Notably, the vertical axis advantage persists regardless of concurrent (phonological, visuospatial, or executive) interference and independently of individual differences in span size, leading to the conclusion that vertical symmetry enhances memory in an automatic fashion (RossiArnaud et al., 2006, Rossi-Arnaud et al., 2012), whereas horizontal and diagonal symmetry seem to require more effortful processing. Similarly, in visual symmetry detection tasks, vertical symmetry is the easiest to detect (i.e., most salient), followed by horizontal and then oblique orientations (e.g., Herbert \& Humphrey, 1996, Locher \& Wagemans, 1993, Wagemans, Van Gool, \& d'Ydewalle, 1992, Wenderoth, 1994). This may be related to the fact that many objects of interest in the visual world exhibit vertical symmetry, such as human faces and bodies and 
other natural (e.g., animals) and nonliving (e.g., buildings, tools) objects.

Symmetry also aids tactile judgments of shapes and memory for haptically explored configurations. For instance, Ballesteros, Millar, and Reales (1998) found that symmetry facilitated tactile processing when participants had to judge whether raised-line shapes were open or closed. We previously reported (Cattaneo et al., 2010) that in a task requiring memorization of the position of a number of cells on haptically explored 2-D configurations, blindfolded sighted participants remembered symmetric configurations better than asymmetric ones. Moreover, as in the visual modality (Rossi-Arnaud et al., 2006, Rossi-Arnaud et al., 2012), vertically symmetric patterns were remembered better than horizontally symmetric ones. Notably, though, early blind participants tested in the same task recalled vertically and horizontally configurations equally well (Cattaneo et al., 2010). We interpreted these findings as suggesting that the vertical symmetry advantage for haptic spatial memory observed in blindfolded sighted participants is likely to have a visual origin. There is evidence that sighted individuals usually adopt a visual translational process when attempting to identify stimuli by touch (e.g., Afonso et al., 2010, Lacey, Flueckiger, Stilla, Lava, \& Sathian, 2010, Scocchia, Stucchi, $\&$ Loomis, 2009). It is thus possible that the sighted generate a visual representation of the explored patterns in which vertical symmetry "pops out" as being most salient.

Our previous findings do not clarify whether the memory advantage for vertical symmetry is lacking in the early blind because of the congenital absence of sight or whether a prolonged deprivation of vision per se can also lead to similar effects. To clarify this issue, in this follow-up study, we investigated the ability of late blind individuals to remember spatial configurations that were either vertically or horizontally symmetric or asymmetric (using the same task as that described in Cattaneo et al., 2010). In contrast to the congenitally or very early blind, late blind individuals possess previous visual experiences and memories. This study was designed to disambiguate the possibility that early visual experience mediates the vertical symmetry advantage in a memory task.

To permit direct comparison with the results of our previous study (Cattaneo et al., 2010), patterns were presented in both a horizontal (lying on a table) and a frontal (perpendicular to the line of sight) plane. We originally introduced this manipulation expecting that vertically symmetric patterns would be remembered better when presented frontally than when presented horizontally, given that vertical symmetry is more typically perceived in the frontal plane (e.g., faces, buildings). Although we found that plane of placement did not affect performance in either the sighted or early blind (Cattaneo et al., 2010), this experimental manipulation remains potentially important. There is evidence that given a spatial reference frame (e.g., one based on environmental cues, such as gravity or on perceiver position, or even a more "intrinsic" frame that is centered on perceived objects; see Carlson, 1999), preferences in haptic-based tasks vary in blind and sighted participants depending on the (frontal vs. horizontal) plane of presentation (e.g., Gentaz \& Hatwell, 1998, Struiksma, Noordzij, \& Postma, 2011). In the blind, the preference for a particular reference frame may also be related to their haptic experience. Specifically, the blind typically touch and manipulate objects oriented along a horizontal plane (e.g., Braille is normally read on a horizontal surface), whereas exploring in the frontal plane may be less familiar.

\section{Method}

\section{Participants}

Twelve late blind (4 female; mean age, 54.3 years $\pm 6.1 S D$ ), and 12 sighted control participants (4 female; mean age, 55.5 years $\pm 6.0 S D$ ) took part in the experiment. All the late blind participants had documented profound vision loss and were all proficient Braille readers. The mean duration of blindness was 29.8 years $\pm 14.6 S D$, and mean age of blindness onset was 24.4 years $\pm 14.0 S D$. All causes of blindness were of ocular origin and were not associated with a central neurological disorder. Table 1 summarizes the characteristics of the blind participants. All participants were right-handed (Oldfield, 1971). A local ethics committee approved the experiment.

\section{Materials}

The materials and procedure were the same as those used to test early blind individuals (see Cattaneo et al., 2010). This memory matrix task has been used in previous studies testing memory span in blind and visually impaired participants (e.g., Cattaneo \& Vecchi, 2008). Briefly, tactile stimuli were two-dimensional wooden matrices measuring approximately $20 \mathrm{~cm}$ a side and consisting of 25 perceivable cells. Each cell in the matrix measured $4 \mathrm{~cm}$ a side and was $2 \mathrm{~mm}$ high, as compared with the matrix plane. The gap distance between each cell was $5 \mathrm{~mm}$. Tactile differences were created by covering 7 cells with sandpaper ( 220 grit and covering the entire surface of the cell) on each trial. Matrices were presented in both a horizontal and a frontal plane. In the horizontal plane condition, matrices were presented on a table. The center of the matrix was aligned with the participant's body midline, and the bottom side of the matrix was placed at a distance of about $20 \mathrm{~cm}$ away from the participant. In the frontal plane, the matrix was aligned 
Table 1 Characteristics of late blind participants

\begin{tabular}{lllllll}
\hline Participant & Sex & $\begin{array}{l}\text { Age } \\
\text { (years) }\end{array}$ & $\begin{array}{l}\text { Years of } \\
\text { education }\end{array}$ & Blindness etiology & $\begin{array}{l}\text { Age of } \\
\text { onset }\end{array}$ & Level of visual function \\
\hline 1 & M & 58 & 16 & retinal detachment & 5 & NLP \\
2 & M & 49 & 13 & retinitis pigmentosa & 11 & light perception left eye only \\
3 & M & 56 & 15 & retinitis pigmentosa & 5 & light perception \\
4 & F & 63 & 15 & retinal detachment & 23 & NLP \\
5 & M & 53 & 17 & retinal detachment & 24 & NLP \\
6 & M & 60 & 10 & congenital glaucoma and retinal & 38 & NLP \\
7 & M & 57 & 17 & octachment & 46 & light perception (shadows) \\
8 & F & 50 & 10 & retinitis pigmentosa & 40 & NLP \\
9 & M & 61 & 17 & retinitis pigmentosa & 25 & NLP \\
10 & M & 47 & 14 & bilateral optic nerve trauma & 35 & light perception (shadows and hand \\
11 & F & 54 & 11 & retinitis pigmentosa & 30 & motion) \\
12 & F & 43 & 19 & light perception
\end{tabular}

Note. NLP: no light perception

with the participant's body midline. The bottom side of the vertical matrix was fixed at a height of about $10 \mathrm{~cm}$ above the table (a wooden panel was used to hold the matrices) at a distance of $20 \mathrm{~cm}$ away from the participant. Configurations were not made of any movable components, but different panels were created with fixed configurations so that the entire configuration was swapped on each trial. Three configurations were tested: symmetric along the vertical axis (VS), symmetric along the horizontal axis (HS) and nonsymmetric (NS). Examples of the three different types of configurations and testing conditions are shown in Fig. 1. For matrices presented in the frontal plane, the vertical axis of symmetry was in the direction of the force of gravity (i.e., parallel to the participants' midline axis), and the horizontal axis of symmetry was perpendicular to that (i.e., parallel to
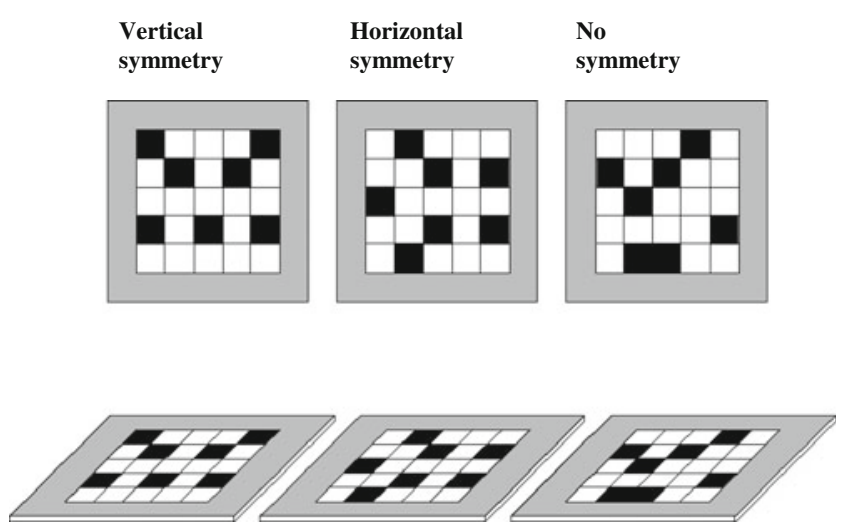

Fig. 1 A schematic representation of the three types of tactile stimuli configurations used in the study (from left to right: vertically symmetric, horizontally symmetric, and asymmetric). Stimuli were presented in the frontal (upper panel) and horizontal (tabletop; lower panel) plane conditions the horizon). When the matrices were presented in the horizontal plane, the vertical and horizontal axes of orientation referred to the horizontal tabletop. That is, the vertical axis of symmetry was in direct vertical (tabletop projected) alignment with the midpoint of the participants' body (i.e., perpendicular to the horizon), and the horizontal axis of symmetry was parallel to the horizon (see Ballesteros et al., 1998).

\section{Procedure}

Participants were seated comfortably at a table. Sighted participants were blindfolded throughout the entire experiment. One blind participant (see Table 1) could perceive the movement of her hand and was, therefore, blindfolded. Although some of the other blind participants had some residual light perception (e.g., locating a light source in the dark; see Table 1 for details), none of them could perceive shape or contrast and, thus, visually distinguish the matrices. For this reason, the blindfold was not used. The experimenter positioned the participants' hands in the middle of the matrix (parallel to the midbody axis) as the starting position on each trial. Participants were instructed to tactilely explore the matrices and to memorize the positions of the target cells. Participants were unaware that symmetry was being investigated in the study. The exploration phase lasted $16 \mathrm{~s}$, recorded by the experimenter. A chronometer was used to time the duration of the exploration, and the end of the exploration time was signaled by a "beep." The recall phase followed the exploration phase, with an approximate delay of 1-2 s needed to replace the target matrix with the response matrix. Before starting the experiment, participants 
were told that they could explore the matrices using one hand or two hands, and in any order they preferred (i.e., scanning direction). The only limitation was the fixed exploration time of $16 \mathrm{~s}$. At testing, participants were required to indicate (by pointing with their right index finger) the positions of the memorized target cells on a corresponding blank matrix. There was no time limit for responding. For each plane (horizontal and frontal), there were 6 trials for the three types of configurations (VS, HS, NS), for a total of 36 trials. The matrices in the horizontal and frontal planes were presented in separate blocks (order was counterbalanced across participants). VS, HS, and NS matrices in each block were presented in random order. The experiment started with 4 practice trials (not included in the analyses), 2 in the horizontal and 2 in the frontal plane conditions. The experiment took approximately $45 \mathrm{~min}$ to complete.

\section{Results}

Figure 2 shows performance accuracy (percentage of correctly remembered cells) for late blind and sighted participants for each configuration and in the horizontal and

\section{a}

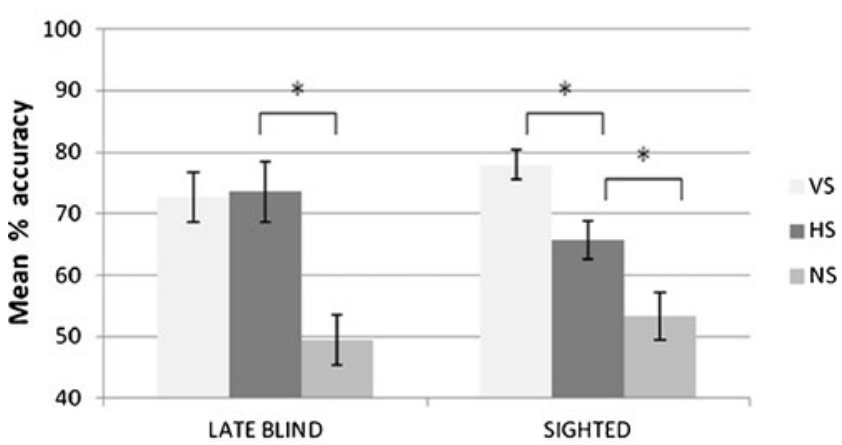

b
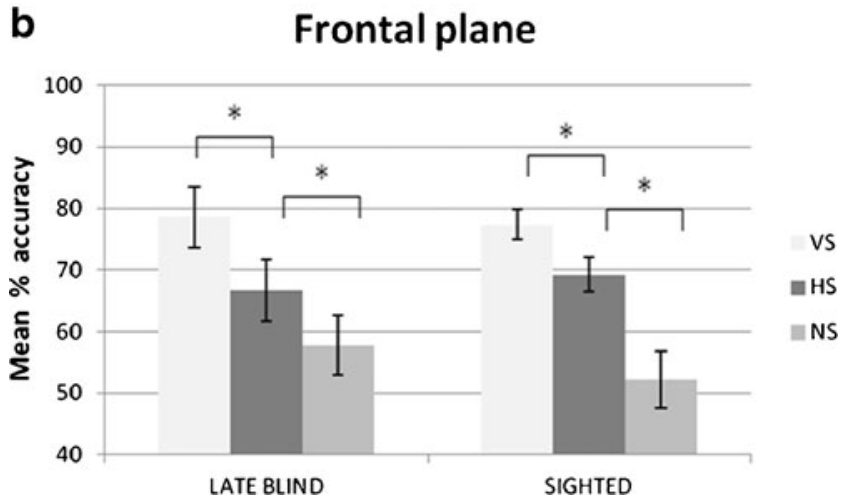

Fig. 2 Mean percentage accuracy of late blind and blindfolded sighted individuals with configurations symmetric along the vertical axis (VS), horizontal axis (HS), and asymmetric (NS) configurations. Results are shown for the horizontal (a) and frontal (b) plane conditions. Error bars represent SEMs. * $p<.05$ frontal plane conditions. A repeated measures ANCOVA was carried out on accuracy (percentage of correctly remembered cells) with configuration (VS, HS, NS) and plane (horizontal vs. frontal) as within-subjects variables, group (late blind vs. sighted) as a between-subjects variable, and participants' age as a covariate. Age was added as a covariate in light of previous evidence suggesting that sensitivity to visual bilateral symmetry may decline with increasing age (Herbert, Overbury, Singh, \& Faubert, 2002). Blindness duration was also considered as an additional covariate in the late blind group.

The analysis revealed a significant effect of configuration, $F(2,42)=55.59, p<.01, \eta_{\mathrm{p}}^{2}=.73$, with symmetric configurations being remembered better than nonsymmetric ones overall, with an additional advantage for VS over HS configurations. The significant three-way plane $\times$ configuration $\times$ group interaction, $F(2,42)=3.58, p=.037, \eta_{\mathrm{p}}{ }^{2}=$ .15 , suggested that this pattern depended on the plane of presentation and on the visual status of participants (see below). Plane $(p=.52)$ and group $(p=.93)$ were not significant, nor were any of the two-way interactions ( $p$ s $>$ $.05)$. Neither the covariate of age $(p=.27)$ nor blindness duration ( $p=.45$ ) was significant, nor did either significantly interact with any other factors (all $p \mathrm{~s}>.05$ ); thus, they were not considered further in the analyses. Bonferroni correction was applied to all post hoc comparisons.

The significant three-way plane $\times$ configuration $\times$ group interaction was further analyzed by separate ANOVAs carried out for each plane in order to look at possible group differences depending on the type of configuration presented. In the frontal plane, the two groups performed similarly (group $\times$ configuration interaction, $p=.46$ ): Regardless of visual status, memory was better for VS than for both HS $(p<.001)$ and NS $(p<.001)$ configurations and for HS than for NS $(p=.003)$ configurations. In the horizontal plane, the group $\times$ configuration interaction was significant, $F(2,44)=3.31, p=.046, \eta_{\mathrm{p}}{ }^{2}=.13$. As is shown by Fig. 2, this interaction likely depended on late blind participants performing particularly well with HS configurations. Although independent samples $t$-tests failed to find a significant group difference with any configuration- $-\mathrm{NS}$ $(p=.49)$, HS $(p=.18)$, and VS $(p=.27)$-additional analyses showed that whereas sighted participants remembered VS better than HS configurations, $t(11)=4.67, p=$ .001 , the vertical advantage was absent in the late blind, $t$ $(11)=0.20, p=.85$.

The exploratory strategies adopted by participants were also considered. Overall, the majority of participants (regardless of visual status) used two hands for exploration and started scanning from the upper row of the matrix, keeping the two hands approximately parallel, then descending down to the next row. Notably, using one hand rather than two resulted in worse memory 
recall, and it was more detrimental for remembering symmetric (especially VS) versus nonsymmetric configurations (see Appendix for details).

\section{Discussion}

Symmetric patterns were remembered better than asymmetric ones, regardless of participants' visual status and plane of placement. This finding confirms previous evidence indicating that symmetry, even if incidentally encoded, works as a principle of Gestalt organization, reducing memory load and, hence, aiding memory performance (Cattaneo et al., 2010, Rossi-Arnaud et al., 2006, Rossi-Arnaud et al., 2012). An additional memory advantage for the vertical axis of symmetry over the horizontal one was observed in blindfolded sighted participants, supporting previous findings (Cattaneo et al., 2010, Rossi-Arnaud et al., 2006, RossiArnaud et al., 2012). The vertical axis advantage was also present in the late blind, but only when configurations were presented in the frontal plane. Conversely, when patterns were presented in the horizontal plane (i.e., lying on a table), the vertical axis memory advantage was not present. This finding for the horizontal plane did not depend on vertically symmetric configurations being remembered less well by late blind, as compared with the sighted, participants. Rather, blind participants seemed to be particularly efficient in this condition in retrieving horizontally symmetric configurations.

We previously showed that early blind individuals remembered symmetric configurations better than asymmetric ones, indicating that symmetry facilitated grouping, reducing memory load. However, they did not show an advantage for vertical over horizontal bilateral symmetry, as was the case for blindfolded sighted participants (Cattaneo et al., 2010). We thus speculated that the additional memory advantage for vertical symmetry was rooted in its visual salience. Here, we show that prior visual experience is sufficient to produce a vertical symmetry advantage, but only when configurations are presented in the frontal plane. These findings can possibly be explained by considering the influence of the reference frame participants adopted during exploration.

It is likely that in the frontal plane, an "absolute" reference (i.e., related to the environment; see Carlson, 1999) was predominant regardless of visual status. In fact, previous evidence with sighted and blind participants has shown that in haptic exploration in the frontal plane, geocentric cues (i.e., the direction of the pull of gravity) offer the most powerful frame of reference (see Gentaz \& Hatwell, 1996, 1998). Accordingly, it is possible that vertical symmetry became more salient in the vertical plane due to the additional "reinforcement" (or priming) by the external vertical frame of reference (coinciding with the lateral sides of the matrices) (see Wagemans, 1992). In line with this, it has been shown that the external frame may either reinforce symmetry detection when frame and axis of symmetry are aligned or interfere with symmetry detection in the case of a misalignment between the two. For instance, Herbert, Humphrey, and Jolicoeur (1994) showed that a tilted frame eliminated the orientation bias for vertical symmetry, producing a situation where vertical and tilted symmetry were detected equally fast.

In the horizontal plane, "verticality," even if skewed, was still the main reference for sighted individuals. Conversely, late blind individuals seemed to prefer an "intrinsic" (see Carlson, 1999) reference frame - in the case of our task, referring to the spatial disposition of the target cells with respect to each other in which the internal horizontal axis of symmetry gained importance. This finding is in line with previous results reported by Struiksma et al. (2011), where blindfolded sighted and blind participants (late and early blind were considered as a single group) were tested on a sentence verification task assessing which reference frame is preferred when spatial language is matched to the haptic domain. When objects whose reciprocal spatial relations (e.g., "above," "in front") had to be judged were presented in the frontal plane, the vertical gravity cue made the external reference frame the dominant one regardless of visual status. Conversely, when objects were presented on a horizontal surface, the blind referred more to the "intrinsic" (functional) relation between objects.

This explanation would also account for the pattern of results we previously obtained with the early blind (Cattaneo et al., 2010). In that case, the early blind did not show a preference for vertical symmetry even in the frontal plane, possibly due to their preference for an intrinsic reference frame regardless of stimulus placement. The sense of frontal plane "verticality" may be less pronounced in the early blind, given that they have never been visually exposed to vertically symmetric objects. Conversely, late blind individuals have exposure and experience with the visual world for many years prior to losing their sight. We therefore speculate that this visual "imprinting" has made late blind individuals more sensitive to the external environment reference frame when exploring objects in the frontal plane (in which vertical symmetry is usually salient). In this regard, it is worth noting that blind individuals are taught to use touch to explore objects as well as read Braille (note that all our blind participants were Braille readers), typically on a tabletop surface (i.e., horizontal plane). Therefore, we may speculate that visual imagery (and hence, reference to the rules of the visual world, such as the preferential occurrence of vertical symmetry in the frontal plane) becomes more important when late blind individuals explore objects in the frontal plane, where they lack haptic familiarity. In fact, 
visual imagery has been found to play a critical role in mediating tactile perception and recognition in normally sighted individuals (see Lacey et al., 2010, Scocchia et al., 2009), as also is suggested by the involvement of the occipital cortex in haptic discrimination (e.g., Merabet et al., 2007, Merabet et al., 2004). Accordingly, different studies suggest that prior visual experience can be used by the late blind to support their haptic and auditory perception, thereby helping them to generate a mental representation of the surrounding environment (e.g., Alary et al., 2008, Postma, Zuidhoek, Noordzij, \& Kappers, 2008, Vanlierde \& WanetDefalque, 2004).

In the present study, late blind and blindfolded sighted participants showed an overall similar level of performance, whereas for the same task, early blind participants outperformed their sighted peers in our previous work (Cattaneo et al., 2010). Previous studies also have reported higher performance in early blind, as compared with both late blind and sighted, individuals in haptic tasks, thanks to their superior haptic experience possibly mediated by compensatory neuroplastic changes (for a review, see Cattaneo et al., 2008). Nonetheless, recourse to visual imagery may facilitate performance in the late blind. Late blind individuals have even been found to perform better than both blindfolded sighted controls and congenitally blind individuals in haptic picture recognition, thanks to the combined advantage of increased haptic skills and the impact of prior experience with pictures (e.g., Heller, 1989, 2003). Hence, the level of performance of early and late blind participants, as compared with sighted participants, is likely to depend on the specific task at play and, specifically, on the extent to which this may call visual processes into play.

Finally, previous evidence suggests that the detection of symmetry improves when two hands (rather than one) are used for tactile exploration (Ballesteros, Manga, \& Reales, 1997, Ballesteros \& Reales, 2004). This was also the case for congenitally blind participants (Cattaneo et al., 2010) and for the late blind participants and sighted control participants tested here. In fact, bimanual exploration allows one to relate hand locations to the body axis, and this provides an effective frame of reference for coding bilateral features of objects (see also Millar, 1978). Moreover, bimanual exploration has been found to benefit performance also in tasks requiring blindfolded sighted participants to recognize tactile images of familiar objects (Wijntjes, van Lienen, Verstijnen, \& Kappers, 2008) and in shape discrimination or drawing tasks tested in both sighted and blind individuals (e.g., Russier, 1999, Vinter, Fernandes, Orlandi, \& Morgan, 2012). Previous evidence has shown that the blind tend to employ both hands and use multiple strategies in haptic recognition more often than do their sighted peers (Rovira, Deschamps, \& Baena-Gomez, 2011; see also Heller, 1989). This may also be a consequence of the specific training the blind receive, on the basis of the finding that bimanual exploration enlarges the tactile field perceived and, thus, enhances tactile/haptic performance (Loomis, Klatzky, \& Lederman, 1991). In our study, bimanual exploration was spontaneously adopted as the main strategy by the majority of participants, regardless of their visual status. It is likely that the particular task induced the adoption of the more effective exploratory strategy in sighted participants as well, with different haptic exploratory strategies being suitable for providing information about different object properties (such as shape, size, or texture) (cf. Lederman \& Klatzky, 2009).

In summary, our findings suggest that symmetry overall works as a Gestalt principle of spatial organization across sensory modalities. Critically, having prior visual experience is sufficient to determine the emergence of a memory advantage for vertical, as compared with horizontal, symmetry, but only for frontally presented patterns. This may depend on vertical symmetry usually appearing in the frontal plane in the visual world.

Acknowledgments We would like to thank Professor Simon Grondin, Professor Johan Wagemans, and two anonymous reviewers for their valuable comments on our manuscript.

\section{Exploratory strategies}

On each trial, the spontaneous exploratory strategy adopted by each participant was noted by the experimenter. For the purposes of the study, the experimenter recorded whether the participant spontaneously used one hand or two hands for the exploration and the direction in which the matrix was explored (e.g., starting from the upper row, parallel vs. sequential search, etc.). Again, no explicit exploring strategy was proposed to the participant prior to participating in the study.

Overall, late blind participants were quite consistent in their exploration strategies. In particular, 8 out of the 12 blind participants used two hands in exploring the matrices on all trials, regardless of the plane of presentation. One participant (participant 4) used only her right hand to explore the matrices, keeping her left hand anchored to the left side of the matrix. Two participants (participants 9 and 10) used both hands on all the trials in the frontal plane, while in the horizontal plane, these participants kept their left hand anchored to the left side of the matrix and explored the matrix with their right hand only (both of these participants first performed the task in the frontal plane and then in the horizontal plane). Another participant (participant 6) did the opposite of the latter 2 participants, using two hands on all the trials for the horizontal plane and only the right hand on all the trials for the frontal plane (this participant first performed the task in the horizontal plane and then in the frontal plane). 
Notably, memory performance of the 3 blind participants who explored with one hand in the horizontal plane tended to be poorer, as compared with the mean accuracy of the other blind individuals who explored with two hands, being equal to $61.90 \%$ for vertically symmetric (VS) configurations (vs. $76.19 \%$ for the other 9 blind participants), $t(10)=$ $1.66, p=.13 ; 57.94 \%$ for horizontally symmetric (HS) configurations (vs. $78.84 \%$ for the other 9 blind participants), $t(10)=2.10, p=.062$; and $38.10 \%$ for nonsymmetric (NS) configurations (vs. $53.18 \%$ for the other 9 blind participants), $t(10)=1.33, p=.21$. Similarly, memory performance of the 2 blind participants who explored with one hand in the frontal plane tended to be poorer, as compared with the mean accuracy of the other blind individuals who explored with two hands, their accuracy being equal to $53.58 \%$ for VS configurations (vs. $83.57 \%$ for the rest of the group), $t(10)=2.95, p=.014 ; 48.81 \%$ for HS configurations (vs. $70.24 \%$ for the rest of the group), $t(10)=1.73$, $p=.11$; and $52.38 \%$ for NS configurations (vs. $58.81 \%$ for the rest of the group), $t(10)=0.47, p=.65$. Notably, for both the horizontal and the frontal planes, using one hand was more detrimental for remembering symmetric versus asymmetric configurations. Critically, when comparing memory accuracy for VS and HS configurations in the two planes for only the 8 blind participants who consistently used two hands in both the horizontal and frontal planes, we replicated the same pattern of results as that found when all blind participants were included, regardless of their exploration strategy: VS configurations were remembered better than HS configurations in the frontal plane, $t(7)=5.38, p=.001$, but not in the horizontal one, $t(7)=0.29, p=.78$.

Late blind participants also tended to be very consistent in the direction of the first exploration of the matrices throughout the experiment. In particular, 11 blind participants always started scanning from the upper row of the matrix (regardless of the plane of presentation), keeping the two hands approximately parallel, with the left hand exploring the left portion of the row and the right hand exploring the right portion of the row, then descending down to the next row (raising their hands and positioning them at the beginning of the following row) and going down to the bottom row (participants who scanned with one hand followed an upper-left to bottom-right direction similar to a reading-like motion). One blind participant (participant 1) started exploration from the bottom row, in both the frontal and horizontal plane presentation conditions.

Nine sighted participants used two hands on all the experimental trials, regardless of the plane of presentation, following the "up-down search in parallel" strategy described above on all the trials (with the exception of 1 participant, who, in the horizontal plane, followed a bottom-to-top direction of exploration). The remaining 3 participants used only the right hand on all trials, while keeping the left hand anchored to the left side of the matrix. Two of the 3 sighted participants who scanned only with the right hand followed a reading-like strategy of exploration on all the trials (i.e., they started from the upper-left corner; when they arrived at the end of the first row, they raised their hand and restarted from the beginning of the second row, etc.).

The 3 sighted participants who explored with one hand reported a mean percentage of accuracy (collapsed across the two planes) of $72.22 \%$ for VS configurations (vs. $79.50 \%$ for participants who used two hands) and $62.70 \%$ and $51.59 \%$ for HS and NS configurations, respectively (sighted participants who used two hands scored 69.05\%, for HS and $53.17 \%$ for NS matrices). Overall, the use of one hand rather than two seemed to be detrimental to memory accuracy for vertical symmetric configurations only, $t(10)=$ $2.40, p<.05$, whereas it did not significantly affect memory for HS $(p=.26)$ and NS $(p=.84)$ configurations.

\section{References}

Afonso, A., Blum, A., Katz, B. F. G., Tarroux, P., Borst, G., \& Denis, M. (2010). Structural properties of spatial representations in blind people: Scanning images constructed from haptic exploration or from locomotion in a 3-D audio virtual environment. Memory $\&$ Cognition, 38, 591-604.

Alary, F., Goldstein, R., Duquette, M., Chapman, C. E., Voss, P., \& Lepore, F. (2008). Tactile acuity in the blind: A psychophysical study using a two-dimensional angle discrimination task. Experimental Brain Research, 187(4), 587-594.

Ballesteros, S., Manga, D., \& Reales, J. M. (1997). Haptic discrimination of bilateral symmetry in 2-dimensional and 3-dimensional unfamiliar displays. Perception \& Psychophysics, 59(1), 37-50.

Ballesteros, S., Millar, S., \& Reales, J. M. (1998). Symmetry in haptic and in visual shape perception. Perception \& Psychophysics, 60 (3), 389-404.

Ballesteros, S., \& Reales, J. M. (2004). Visual and haptic discrimination of symmetry in unfamiliar displays extended in the z-axis. Perception, 33(3), 315-327.

Carlson, L. A. (1999). 'Selecting a reference frame. Spatial Cognition and Computation, 1, 365-379.

Cattaneo, Z., Fantino, M., Tinti, C., Silvanto, J., Pascual-Leone, A., \& Vecchi, T. (2010). Symmetry perception in the blind. Acta Psychologica, 134(3), 398-402.

Cattaneo, Z., \& Vecchi, T. (2008). Supramodal effects in visual and haptic spatial processes. Journal of Experimental Psychology. Learning, Memory, and Cognition, 34(3), 631-642.

Cattaneo, Z., Vecchi, T., Cornoldi, C., Mammarella, I., Bonino, D., Ricciardi, E., \& Pietrini, P. (2008). Imagery and spatial processes in visual impairments. Neuroscience and Biobehavioral Reviews, 32, 1346-1360.

Gentaz, E., \& Hatwell, Y. (1996). Role of gravitational cues in the haptic perception of orientation. Perception \& Psychophysics, 58, 1278-1292.

Gentaz, E., \& Hatwell, Y. (1998). The haptic oblique effect in the perception of rod orientation by blind adults. Perception \& Psychophysics, 60(1), 157-167.

Heller, M. A. (1989). Picture and pattern perception in the sighted and the blind: The advantage of the late blind. Perception, 18(3), 379389. 
Heller, M. (2003). Superior haptic perceptual selectivity in late-blind and very low-vision subjects. Perception, 32, 499-511.

Herbert, A. M., \& Humphrey, G. K. (1996). Bilateral symmetry detection: Testing a 'callosal' hypothesis. Perception, 25(4), 463-480.

Herbert, A. M., Humphrey, G. K., \& Jolicoeur, P. (1994). The detection of bilateral symmetry: Effects of surrounding frames. Canadian Journal of Experimental Psychology, 48, 140-148.

Herbert, A. M., Overbury, O., Singh, J., \& Faubert, J. (2002). Aging and bilateral symmetry detection. Journal of Gerontology: Psychological Sciences, 57B, P241-P245.

Lacey, S., Flueckiger, P., Stilla, R., Lava, M., \& Sathian, K. (2010). Object familiarity modulates the relationship between visual object imagery and haptic shape perception. NeuroImage, 47, 19771990.

Lederman, S. J., \& Klatzky, R. L. (2009). Haptic perception: A tutorial. Attention, Perception, \& Psychophysics, 71, 1439-1459.

Locher, P. J., \& Wagemans, J. (1993). Effects of element type and spatial grouping on symmetry detection. Perception, 22, 565-587.

Loomis, J. M., Klatzky, R. L., \& Lederman, S. J. (1991). Similarity of tactual and visual picture recognition with limited field of view. Perception, 20(2), 167-177.

Merabet, L. B., Swisher, J. D., McMains, S. A., Halko, M. A., Amedi, A., Pascual-Leone, A., \& Somers, D. C. (2007). Combined activation and deactivation of visual cortex during tactile sensory processing. Journal of Neurophysiology, 97(2), 1633-1641.

Merabet, L. B., Thut, G., Murray, B., Andrews, J., Hsiao, S., \& Pascual-Leone, A. (2004). Feeling by sight or seeing by touch? Neuron, 42(1), 173-179.

Millar, S. (1978). Aspects of memory for information from touch and movement. In G. Gordon (Ed.), Active touch: The mechanism of recognition of objects by manipulation: A multidisciplinary approach (pp. 215-227). Oxford: Pergamon Press.

Oldfield, R. C. (1971). The assessment and analysis of handedness: The Edinburgh inventory. Neuropsychologia, 9, 97-113.

Postma, A., Zuidhoek, S., Noordzij, M. L., \& Kappers, A. M. (2008). Haptic orientation perception benefits from visual experience: Evidence from early-blind, late-blind, and sighted people. Perception \& Psychophysics, 70(7), 1197-1206.
Rossi-Arnaud, C., Pieroni, L., \& Baddeley, A. (2006). Symmetry and binding in visuo-spatial working memory. Neuroscience, 139(1), 393-400.

Rossi-Arnaud, C., Pieroni, L., Spataro, P., \& Baddeley, A. (2012). Working memory and individual differences in the encoding of vertical, horizontal and diagonal symmetry. Acta Psychologica, 56(2), 157-166.

Rovira, K., Deschamps, L., \& Baena-Gomez, D. (2011). Mental rotation in blind and sighted adolescents: The effects of haptic strategies. European Review of Applied Psychology, 61, 153-160.

Russier, S. (1999). Haptic discrimination of two-dimensional raisedline shapes by blind and sighted adults. Journal of Visual Impairment and Blindness, 93, 421-426.

Scocchia, L., Stucchi, N. A., \& Loomis, J. M. (2009). The influence of facing direction on the haptic identification of two-dimensional raised pictures. Perception, 38(4), 606-612.

Struiksma, M. E., Noordzij, M. L., \& Postma, A. (2011). Reference frame preferences in haptics differ for the blind and sighted in the horizontal but not in the vertical plane. Perception, 40(6), 725738.

Vanlierde, A., \& Wanet-Defalque, M. C. (2004). Abilities and strategies of blind and sighted subjects in visuo-spatial imagery. Acta Psychologica, 116(2), 205-222.

Vinter, A., Fernandes, V., Orlandi, O., \& Morgan, P. (2012). Exploratory procedures of tactile images in visually impaired and blindfolded sighted children: How they relate to their consequent performance in drawing. Research in Developmental Disabilities, 33(6), 1819-1831.

Wagemans, J. (1992). Perceptual use of nonaccidental properties. $\mathrm{Ca}$ nadian Journal of Psychology, 46, 236-279.

Wagemans, J., Van Gool, L., \& d'Ydewalle, G. (1992). Orientational effects and component processes in symmetry detection. Quarterly Journal of Experimental Psychoogy, 44A, 475-508.

Wenderoth, P. (1994). The salience of vertical symmetry. Perception, 23(2), 221-236.

Wijntjes, M. W. A., van Lienen, T., Verstijnen, I. M., \& Kappers, A. M. L. (2008). Look what I have felt: Unidentified haptic line drawing are identified after sketching. Acta Psychologica, 128, 255-263. 